\title{
Análise experimental da adição de fibras poliméricas nas propriedades mecânicas do concreto
}

\section{Experimental analyses of polymeric fiber addition on concrete mechanical properties}

\footnotetext{
${ }^{1}$ Departamento de Engenharia de Materiais - POSMAT/CEFET-MG, Belo Horizonte -MG e-mail: josueamaraljr@gmail.com

${ }^{2}$ Departamento de Engenharia de Civil - DEC/CEFET-MG, Belo Horizonte -MG

e-mail: luiza_caroline18@hotmail.com; moravia@civil.cefetmg.br
}

\section{RESUMO}

A adição de fibras ao concreto é uma prática que busca reduzir o surgimento de fissuras e suas respectivas aberturas. Diversos tipos de fibras são utilizadas com esse fim como, por exemplo, as fibras de aço e as fibras poliméricas. O presente trabalho tem como objetivo avaliar a influência da adição de fibras poliméricas nas propriedades mecânicas do concreto. Para isso foram realizados ensaios de resistência à compressão, módulo de elasticidade, resistência à tração na flexão e avaliada a tenacidade de corpos de prova confeccionados com concreto convencional (sem adição de fibras), concreto com adição de fibras de polipropileno e concreto com adição de fibras de polietileno de alto módulo. O comprimento das fibras poliméricas utilizadas é de $12 \mathrm{~mm}$. Os resultados mostram que essas adições melhoram o desempenho do concreto para a resistência à tração e tenacidade, porém não causaram variações significativas na resistência à compressão e no módulo de elasticidade.

Palavras-chave: concreto, fibra, fibras poliméricas, polipropileno, polietileno

\section{ABSTRACT}

The addition of fibers to concrete is a practice that seeks to reduce the appearance of cracks and their respective openings. Various types of fibers are used for this purpose, for example, steel fibers and polymeric fibers. This study evaluated the influence of the addition of fibers on the mechanical properties of concrete. For that were performed compressive strength tests, modulus of elasticity, tensile strength in bending and evaluated the tenacity of specimens made with conventional concrete (without fibers), concrete with addition of polypropylene fibers and concrete with adding high modulus polyethylene fibers. The results show that the additions improved concrete performance to tensile strength and toughness, but did not cause significant variations in the compressive strength and modulus of elasticity.

Keywords: concrete, fiber, polymeric fiber, polypropylene, high modulus polyethylene.

\section{INTRODUÇÃO}

O concreto consiste em um material compósito com propriedades típicas de materiais cerâmicos como por exemplo a fragilidade. Sua composição básica apresenta uma matriz cimentícia, formado por cimento e água, que envolve os agregados graúdos e miúdos. O concreto possui uma resistência à compressão, adequada para o uso na construção civil. No entanto, possui baixos valores para a resistência a tração, o que leva a necessidade de reforçar o material para esse tipo de esforço [1].

A utilização de fibras como adição no concreto tem como principal objetivo reduzir o surgimento de fissuras e diminuir suas aberturas. Esse tipo de reforço ganhou muito interesse na indústria da construção e por parte de pesquisadores a partir da década de 60[2]. 
Diversos tipos de fibras são utilizadas como reforço de concreto, sendo as principais, fibras de aço, fibras poliméricas e fibras naturais. Recentemente diversos estudos sobre a influência das fibras de aço no comportamento concreto foram publicados[3,4,5,6].

A influência das fibras poliméricas no comportamento do concreto foi analisada e estudada por diversos pesquisadores sobre diferentes aspectos $[7,8,9,10]$. A adição de fibras de poliproileno melhora significativamente propriedades mecânicas do concreto, entre elas resistência à tração [9, 11, 12], tenacidade [9] e resistência à abrasão $[9,13]$. A resistência à compressão apresenta pouca ou nenhuma variação com adição de fibras poliméricas $[11,12,14,15]$. O módulo de elasticidade do concreto também mostra não apresenta variação significativa com adição de fibras [16].

O polietileno de alto módulo, conhecido também pela sigla HMPE, oriunda da nomenclatura em inglês high modulus polyethylene, é um dos principais materiais na confecção de cabos submarinos para serviços de ancoragem[17]. Até a data de publicação deste trabalho não foram encontradas estudos a respeito da utilização das fibras de HMPE como adição ao concreto. Está incluso no escopo deste trabalho, avaliar os resultados obtidos para o concreto reforçado com fibras de HMPE, e comparar esses resultados com os obtidos para o concreto com fibras de polipropileno para o concreto sem adição de fibras.

\section{MATERIAIS E MÉTODOS}

$\mathrm{O}$ concreto utilizado neste trabalho apresentou densidade de $2,3 \mathrm{~g} / \mathrm{cm}^{3}$ e foi confeccionado utilizando cimento CP-V-ARI, areia média lavada, brita calcaria tamanho 1, com tamanho máximo de $24 \mathrm{~mm}$ e densidade apararente de $2,4 \mathrm{~g} / \mathrm{cm}^{3}$, água potável, fibras de polipropileno (PP) e fibras de polietileno de alto módulo (HMPE).

O traço utilizado, 1:2:2 com teor a/c de o,61, foi projetado para um fck de $25 \mathrm{MpaA}$ adição de fibras aconteceu no teor de $1 \%$ em relação ao volume da matriz do compósito. A Tabela 1 apresenta as proporções de materiais consumidos para cada tipo de concreto estudado.

Tabela 1: Proporções de materiais utilizados em cada traço estudado.

\begin{tabular}{l|l|l|l|l|l|}
\hline CONCRETO & CIMENTO & AREIA & BRITA & ÁGUA & FIBRAS \\
\hline Sem fibras & $1000 \mathrm{~g}$ & $2500 \mathrm{~g}$ & $2500 \mathrm{~g}$ & $610 \mathrm{ml}$ & $0 \%$ \\
\hline Com fibras PP & $1000 \mathrm{~g}$ & $2500 \mathrm{~g}$ & $2500 \mathrm{~g}$ & $610 \mathrm{ml}$ & $1 \%$ \\
\hline Com fibras HMPE & $1000 \mathrm{~g}$ & $2500 \mathrm{~g}$ & $2500 \mathrm{~g}$ & $610 \mathrm{ml}$ & $1 \%$ \\
\hline
\end{tabular}

As fibras de polipropileno e polietileno de alto módulo utilizadas nesse trabalho possuem o mesmo comprimento, $12 \mathrm{~mm}$. Na etapa de caracterização das fibras, foi possivel estimar o diametro das fibras através do ensaio de microscopia eletronica de varredura. A fibra de polipropileno possui um diâmetro médio de $35 \mu \mathrm{m}$ e a fibra de polietileno de alto módulo um diâmetro médio de $20 \mu \mathrm{m}$. A Figura 1 mostra as fibras utilizadas nesse trabalho e a Tabela 2 apresenta as propriedades de interesse para esse trabalho conforme informações fornecidas pelos fabricantes.

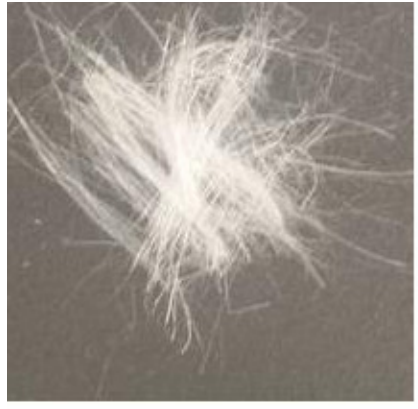

(a)

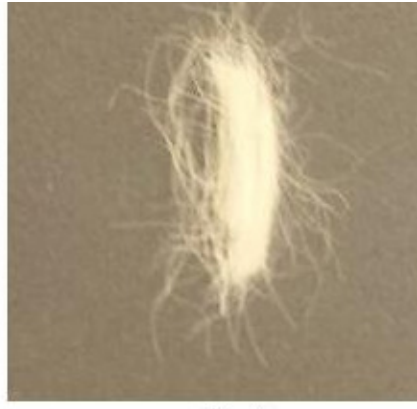

(b)

Figura 1: Fibras poliméricas utilizadas neste trabalho. (a) - Fibra de polietileno de alto módulo; (b) - fibra de polipropileno

Tabela 2: Propriedades das fibras de polipropileno (PP) e polietileno de alto módulo (HMPE). 


\begin{tabular}{l|l|l|l|l|l|}
\hline FIBRA & DENSIDADE & DIAMETRO & $\begin{array}{l}\text { RESITÊNCIA } \\
\text { À TRAÇÃO }\end{array}$ & $\begin{array}{l}\text { MÓDULO DE } \\
\text { ELASTICIDADE }\end{array}$ & ALONGAMENTO \\
\hline Fibra PP & $0,90 \mathrm{~g} / \mathrm{cm}^{3}$ & $35 \mu \mathrm{m}$ & $41,4 \mathrm{MPa}$ & $1,55 \mathrm{GPa}$ & $100-600 \%$ \\
\hline Fibra HMPE & $0,91 \mathrm{~g} / \mathrm{cm}^{3}$ & $20 \mu \mathrm{m}$ & $3000 \mathrm{MPa}$ & $132 \mathrm{GPa}$ & $3,5 \%$ \\
\hline
\end{tabular}

Para avaliar o módulo de elasticidade e a resistência à compressão foi utilizado o método proposto pela norma NBR 8522 [18]. Foram confeccionados 4 corpos de provas cilíndricos, com $10 \mathrm{~cm}$ de diâmetro e $20 \mathrm{~cm}$ de altura, para cada concreto avaliado: concreto convencional (sem fibras), concreto com fibras de PP e concreto com fibras de HMPE. Os corpos de prova passaram por um período de cura úmida de 7 dias. Após esse período eles passaram por um processo de secagem ao ar e foram capeados utilizando argamassa de enxofre fundida.

Os ensaios ocorreram 7 dias após a moldagem utilizando a prensa mecânica do laboratório de mecânica dos pavimentos e tecnologia dos materiais do CEFET-MG / Departamento de Transportes. Para medir a carga aplicada ao corpo de prova foi utilizada uma célula de carga com capacidade máxima de $2000 \mathrm{kN}$. Foram fixados em laterais opostas dos corpos de provas, dois clipes gauges responsáveis por medir as deformações axiais devido ao esforço de compressão.

Para o ensaio de resistência à tração foi adotada a metodologia descrita na norma NBR 12142 [19]. Foram moldados 4 corpos de prova prismáticos para cada tipo de concreto estudado. As dimensões dos corpos de prova foram de $15 \mathrm{~cm}$ x $15 \mathrm{~cm}$ x $50 \mathrm{~cm}$. Após o enchimento, as formas foram levadas para uma mesa vibratória por 20 segundos, para retirar possíveis bolhas formadas no momento da moldagem.

Os corpos de prova foram centralizados em uma base com dois pontos de apoio com uma distância de $45 \mathrm{~cm}$ entre eles. Foram feitas 4 marcações com giz no corpo de prova, a primeira no ponto de apoio do lado esquerdo, e as demais a $15 \mathrm{~cm}, 30 \mathrm{~cm}$ e $45 \mathrm{~cm}$ de distância da primeira marcação. O carregamento foi realizado em 2 pontos na face superior do corpo de prova, nas marcações de $15 \mathrm{~cm}$ e $30 \mathrm{~cm}$ A aplicação da carga ocorreu a uma taxa de 0,9 MPa por minuto, respeitando as diretrizes da norma NBR 12142 [19]. Após a ruptura, foram medidas todas as dimensões dos corpos de prova necessárias para o cálculo da resistência à tração. A saída de ensaio fornece a carga máxima resistida de cada corpo de prova, e através da Equação 1 calculou-se a resistência à tração do material.

$$
F_{c t}=\frac{p \times l}{b \times d^{2}}
$$

Onde:

$\mathrm{F}_{\mathrm{ct}}$ : resistência à tração na flexão $(\mathrm{MPa})$;

$\mathrm{P}$ : carga máxima aplicada $(\mathrm{N})$;

1: distância entre os cutelos de suporte $(\mathrm{mm})$;

b: largura média do corpo de prova na seção de ruptura $(\mathrm{mm})$;

d: altura média do corpo de prova, na seção de ruptura $(\mathrm{mm})$.

A tenacidade é a capacidade do material absorver energia e se deformar até a sua ruptura. O valor da tenacidade pode ser obtido calculando a área formada pela curva carga x deformação e o eixo das abscissas. O cálculo da tenacidade dos materiais estudados nesse trabalho foi realizado utilizando a curva gerada no ensaio de resistência à tração na flexão.

Para todos os resultados, foram realizadas análises estatísticas com o objetivo de garantir a significância dos resultados encontrados. Para isso, para cada grupo de amostras, foram calculados o desvio padrão, variância e coeficiente de variação.

A repetibilidade consiste na capacidade de um mesmo operador obter resultados consistentes utilizando medidores diferentes, e a reprodutibilidade consiste na capacidade de diversos operadores obterem resultados consistentes utilizando um mesmo medidor.

O procedimento adotado para a mistura do concreto do presente trabalho teve como principal objetivo garantir a dispersão homogênea das fibras no concreto. Para isso, as fibras foram inicialmente misturadas com o cimento e agregado miúdo durante 2 minutos na betoneira. Em seguida, $50 \%$ da água foi adicionada, junto com o agregado graúdo. O restante da água foi adicionada aos poucos, enquanto o concreto misturava na betoneira. $\mathrm{O}$ tempo total de mistura foi de 10 minutos. 


\section{RESULTADOS E DISCUSSÕES}

\subsection{Resistência à Compressão}

Os resultados dos ensaios estão expostos na Figura 2. Para cada tipo de concreto avaliado, o gráfico apresenta o valor médio dos resultados de resistência à compressão e os respectivos intervalos de dispersão.

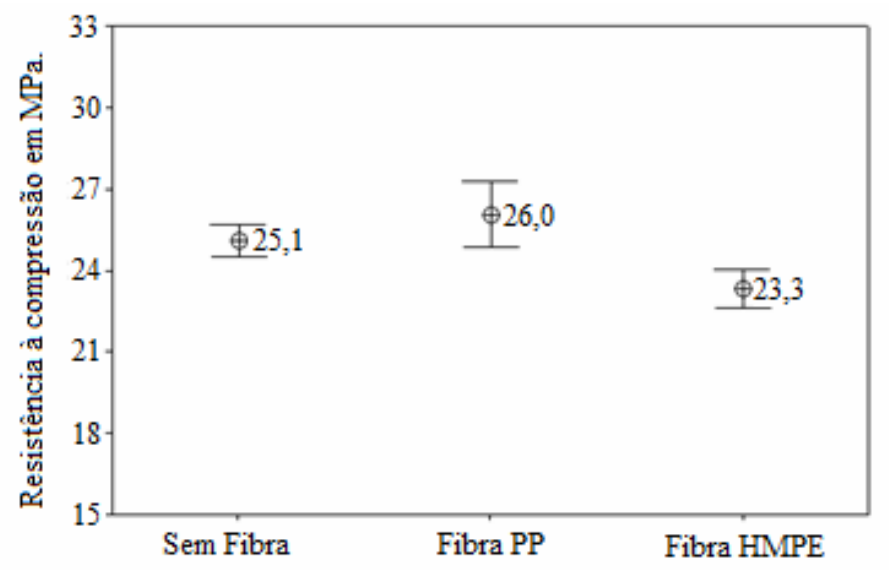

Figura 2: Média e intervalo dos resultado do ensaio de resistência à compressão.

No concreto sem adição de fibras (concreto convencional) obteve-se um valor médio para resistência à compressão de 25,1 MPa. Comparativamente, no concreto com adição de fibras de PP a média obtida foi de 26,0 MPa. Isso representa um acréscimo médio de 3,6\% nos valores de resistência à compressão. Essa variação é muito pequena para ser considerada significativa. Portanto não houve influência da adição de fibras de PP na resistência à compressão do concreto.

O concreto reforçado com fibras de HMPE, o valor médio obtido para a resistência à compressão foi de 23,3 MPa. Isso representa uma redução de 7,2 \% em relação ao concreto sem adição de fibras e redução de $10,4 \%$ em relação ao concreto com fibra de polipropileno. Considerando os valores da variância encontrados para esses grupos de amostras, conclui-se que as fibras de HMPE causaram uma influência negativa na resistência à compressão do concreto. Foram observados nos compôs de prova com fibras de HMPE a presença de nódulos de fibras, que podem ter servido de concentradores de tensões no corpo de prova, contribuindo para essa redução na resistência à compressão.

Ainda no estado fresco, o concreto com fibras de PP apresentou visualmente melhor dispersão das fibras do que o concreto com fibras de HMPE, onde foram encontrados alguns emaranhados.

Neste trabalho, observou-se que a influência das fibras de PP na resistência à compressão do concreto, condiz com as descrições encontradas na literatura.

A partir dos resultados de cada corpo de prova, uma análise estatística foi realizada e está apresentada na Tabela 3. Estando o coeficiente de variação de cada tipo de concreto avaliado, abaixo do limite proposto nesse trabalho, conclui-se que os resultados aqui apresentados para a resistência à compressão são significativos.

Tabela 3: Análise estatística dos resultados do ensaio de resistência à compressão.

\begin{tabular}{c|c|c|c|c|}
\hline CONCRETO & MÉDIA & VARIÂNCIA & DESVIO PADRÃo & COEFICIENTE DE VARIAÇÃO \\
\hline Sem fibra & $25,10 \mathrm{MPa}$ & 0,22 & 0,48 & $1,91 \%$ \\
\hline Fibra PP & $26,00 \mathrm{MPa}$ & 0,43 & 0,97 & $3,73 \%$ \\
\hline $\begin{array}{c}\text { Fibra HM- } \\
\text { PE }\end{array}$ & $23,30 \mathrm{MPa}$ & 0,23 & 0,57 & $2,44 \%$ \\
\hline
\end{tabular}

\subsection{Módulo de Elasticidade}


A Figura 3 apresenta os resultados obtidos nos ensaios de módulo de elasticidade estático para o concreto convencional, concreto com adição de fibra de polipropileno e concreto com adição de fibra de HMPE. Estão presentes nesse gráfico o valor médio do Módulo de Elasticidade de cada corpo de prova e os intervalos de dispersão encontrados.

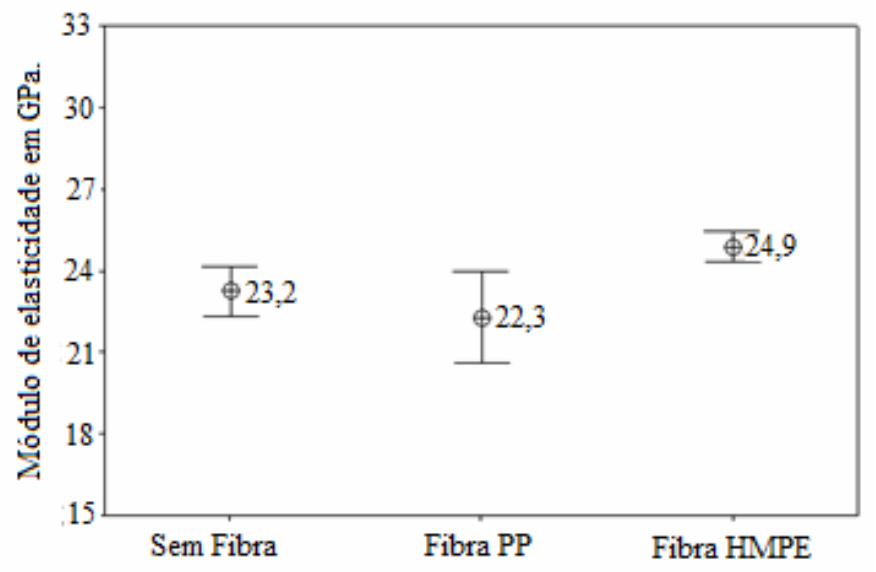

Figura 3: Média e intervalo dos resultado dos ensaio de módulo de elasticidade.

Para o convencional, o valor médio encontrado para o módulo de elasticidade foi de 23,2 GPa. Já o concreto com adição da fibra de polipropileno o valor encontrado para o módulo foi de $22,3 \mathrm{GPa}, 3,9 \%$ menor que o concreto sem fibras.

O concreto com adição de fibras de HMPE apresentou um valor médio de 24,9 GPa, 7,3\% maior do que o concreto com adição de fibras

Nenhuma dessas variações foram consideradas significativas, concluindo que a adição de fibras poliméricas no teor volumétrico de $1 \%$ não afeta o módulo de elasticidade. O mesmo foi verificado na literatura.

A análise estatística dos resultados para módulo de elasticidade está apresentada na Tabela 4. O coeficiente de variação encontrado, para todos os traços de concreto estudados, foram abaixo do limite estabelecidos nesse trabalho para que os resultados sejam significativos.

Tabela 4: Análise estatística dos resultados do ensaio de módulo de elasticidade.

\begin{tabular}{c|c|c|c|c|}
\hline CONCRETO & MÉDIA & VARIÂNCIA & DESVIO PADRÃO & COEFICIENTE DE VARIAÇÃO \\
\hline Sem fibra & $23,2 \mathrm{GPa}$ & 0,34 & 0,75 & $3,23 \%$ \\
\hline Fibra PP & $22,3 \mathrm{GPa}$ & 0,60 & 1,34 & $6,00 \%$ \\
\hline $\begin{array}{c}\text { Fibra HM- } \\
\text { PE }\end{array}$ & $24,9 \mathrm{GPa}$ & 0,20 & 0,44 & $1,76 \%$ \\
\hline
\end{tabular}

\subsection{Resistência à tração na flexão}

Os resultados dos ensaios de resistência à tração na flexão estão apresentados na Figura 4, estando presentes no gráfico a média dos resultados e o intervalo de dispersão. 


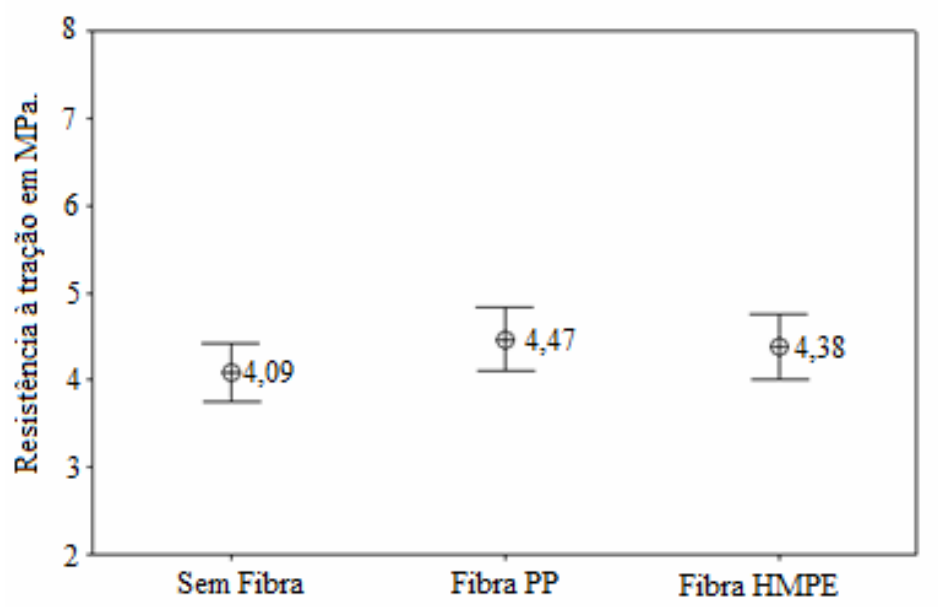

Figura 4: Média e intervalo dos resultado do ensaio de resistência à tração.

$\mathrm{O}$ concreto sem adição de fibras apresentou uma resistência à tração média de 4,09 $\mathrm{MPa}$. $\mathrm{O}$ resultado médio obtido nos ensaios de tração na flexão do concreto com adição de fibras de polipropileno foi de 4,47 MPa enquanto que no concreto com adição de fibras de HMPE o resultado médio foi de 4,38 MPa.

A adição de fibras no concreto levou a um aumento na resistência à tração em relação ao concreto sem fibras. Esse aumento foi de 9,3\% para o concreto com adição de fibras de polipropileno e de 7,1\% para o concreto com adição de fibras de HMPE.

$\mathrm{O}$ aumento da resistência à tração para os concretos com adição de fibras poliméricas em relação ao concreto sem adição de fibras condiz com o encontrado na literatura.

A diferença entre os valores encontrados para o concreto com adição de fibras de polipropileno e para o concreto com fibras de HMPE foi muito pequena, o que deixa claro que apesar da fibra de HMPE possuir propriedades mecânicas superiores as fibras de polipropileno, os nódulos tem um impacto grande na resistência final do material compósito reforçado com fibras.

A análise estatística feita a partir dos resultados de cada corpo de prova está apresentada na Tabela 5.

Tabela 5: Análise estatística dos resultados do ensaio de resistência à tração.

\begin{tabular}{c|c|c|c|c|}
\hline CONCRETO & MÉDIA & VARIÂNCIA & DESVIO PADRÃO & COEFICIENTE DE VARIAÇÃO \\
\hline Sem fibra & $4,09 \mathrm{MPa}$ & 0,11 & 0,21 & $5,13 \%$ \\
\hline Fibra PP & $4,47 \mathrm{MPa}$ & 0,12 & 0,23 & $5,51 \%$ \\
\hline Fibra HMPE & $4,38 \mathrm{MPa}$ & 0,12 & 0,24 & $5,47 \%$ \\
\hline
\end{tabular}

Para os concretos avaliados, o coeficiente de variação ficou abaixo do limite estabelecido nesse trabalho. Isso demonstra a validade dos dados obtidos nos ensaios, e da metodologia adotada.

A Figura 5 apresenta as curvas Carga X Deformação. Cada Grupo apresentado na figura, possui um corpo de prova de cada tipo de concreto estudado. Podemos notar um comportamento um pouco diferente no corpo de prova com adição de fibras de HMPE. O Concreto reforçado com fibras de HMPE deformou mais do que os demais concretos antes da ruptura. 

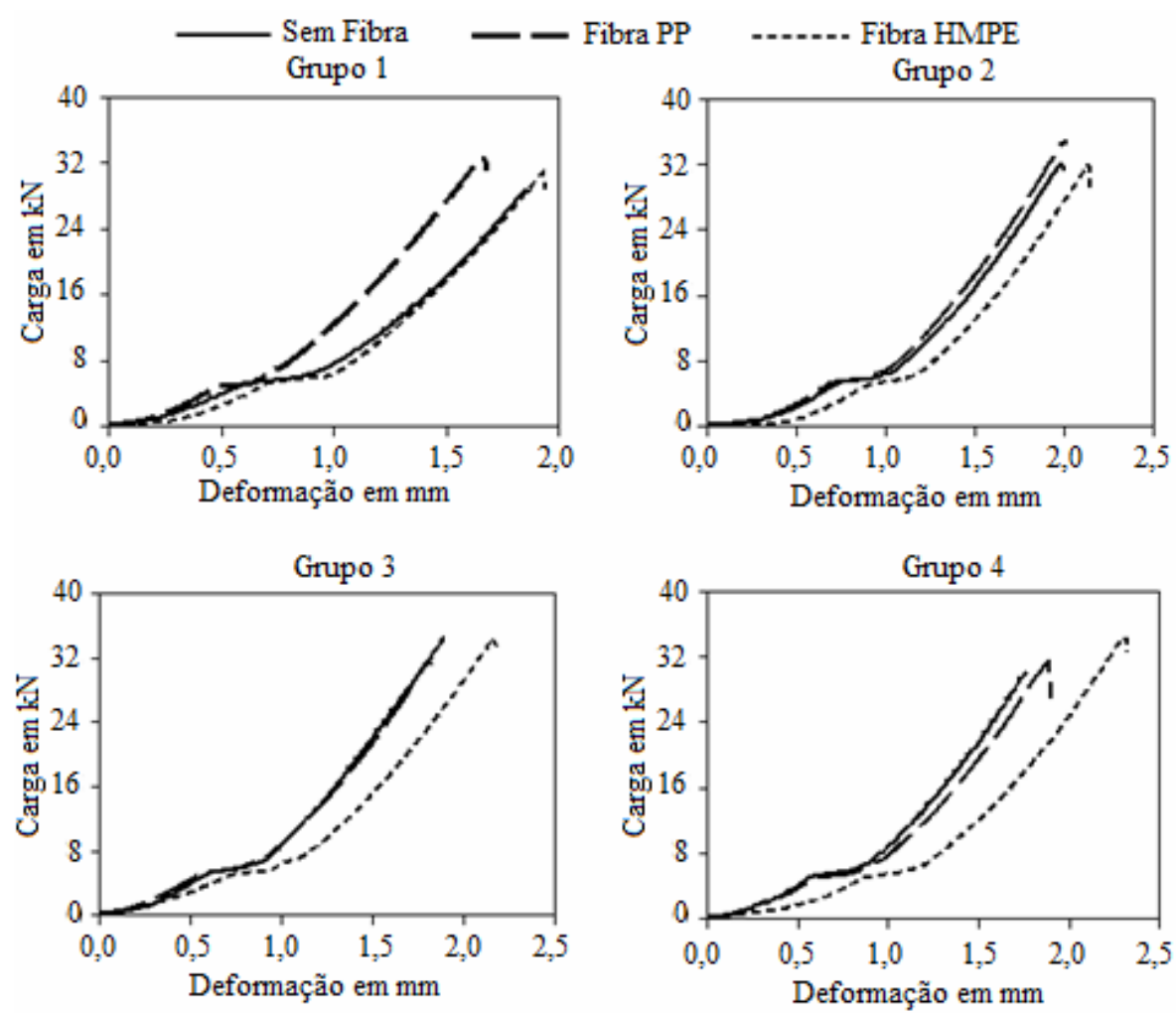

Figura 5: Curvas Carga x Deformação do ensaio de tração na flexão.

\subsection{Tenacidade}

Para obter o valor da tenacidade dos concretos estudados neste trabalho, foi calculada a área embaixo da curva carga $(\mathrm{kN})$ x deformação $(\mathrm{mm})$ obtida no ensaio de resistência à tração na flexão. Para calcular esse área, foi escolhido um método de integração numérica conhecido como integração por retângulos. Os valores médios encontrados para a tenacidade estão ilustrados na Figura 6.

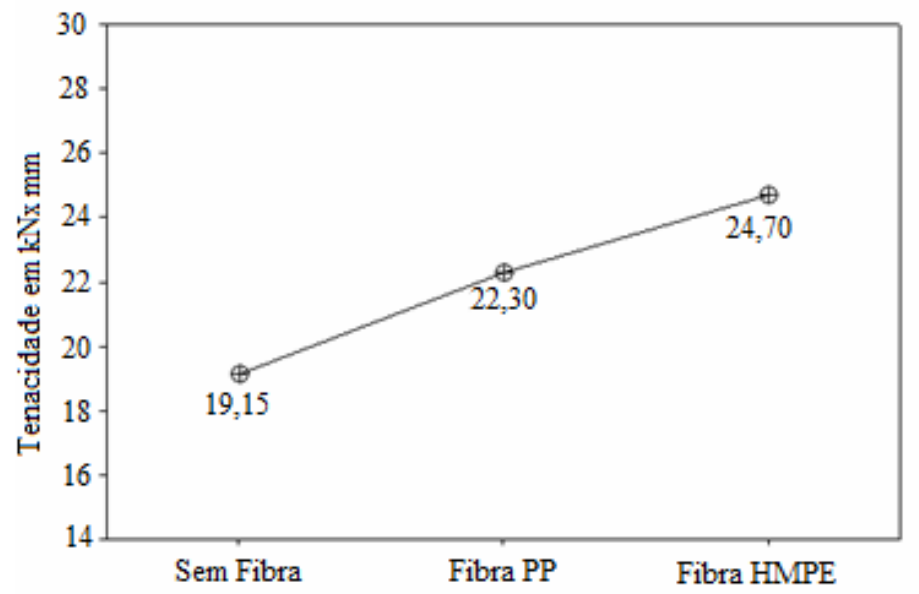

Figura 6: Média e intervalo dos resultado do ensaio de tenacidade.

O concreto sem adição de fibras poliméricas apresentou um valor médio de tenacidade igual a 19,14 kN.mm. Tanto o concreto com adição de fibras de polipropileno quanto o concreto com adição de fibras de HMPE apresentaram valores médios de tenacidade superiores ao concreto sem adição de fibras. No caso do concreto com adição de fibras de polipropileno, a média dos resultados de tenacidade foi de $22,27 \mathrm{kN} . \mathrm{mm}$. 
Isso representa um acréscimo de 16,3 \% em relação ao concreto sem adição de fibras. Já o concreto com adição de fibras de HMPE apresentou uma tenacidade média de 24,72 kN.mm, o que representa uma tenacidade média $29 \%$ maior em relação ao concreto sem fibras. Esses resultados confirmam a hipótese levantada anteriormente de que a adição de fibras poliméricas aumenta a capacidade do concreto de absorver energia antes da ruptura, ou seja, o concreto consegue uma maior deformação até sua ruptura.

Esse comportamento de melhoria significativa da tenacidade do concreto com adição de fibras poliméricas foi constatado também na literatura.

A análise estatística feita em cima dos valores de tenacidade encontrados para cada tipo de concreto estudado está exposta na Tabela 6 . O coeficientes de variação de todos os resultados para tenacidade foi considerado satisfatório por estar abaixo do limite proposto nesse trabalho.

Tabela 6: Análise estatística dos resultados do ensaio de tenacidade.

\begin{tabular}{c|c|c|c|c|}
\hline CONCRETO & MÉDIA & VARIÂNCIA & DESVIO PADRÃO & COEFICIENTE DE VARIAÇÃO \\
\hline Sem fibra & 19,14 kN.mm & 0,55 & 0,95 & $4,9 \%$ \\
\hline Fibra PP & $22,27 \mathrm{kN} . \mathrm{mm}$ & 0,50 & 0,87 & $3,9 \%$ \\
\hline $\begin{array}{c}\text { Fibra HM- } \\
\text { PE }\end{array}$ & $24,72 \mathrm{kN} . \mathrm{mm}$ & 0,08 & 0,12 & $0,5 \%$ \\
\hline
\end{tabular}

\section{CONCLUSÕES}

A investigação experimental realizada neste trabalho com o objetivo de avaliar a influência das fibras poliméricas nas propriedades mecânicas do concreto alcançou as seguintes conclusões:

O concreto com adição de fibras de PP e o concreto com adição de fibras de HMPE apresentaram respectivamente 26,0 MPa e 23,3 MPa para resistência à compressão, enquanto o concreto sem fibras apresentou resistência à compressão de 25,1 MPa. Isso significa que, em relação ao concreto sem fibras, o concreto com fibra de PP apresetnou uma variação de 3,6\% e o concreto com fibra de HMPE uma variação de $7,2 \%$ para os valores de resistência à compressão. Essas variações são pequenas e insignificativas, apontando para o fato de que a adição de fibras poliméricas no teor de $1 \%$ não afeta a resistência à compressão do concreto.

O módulo de elasticidade dos concretos com adição de fibras de PP e adição de fibra de HMPE não sofreu alteração significativa em relação ao módulo do concreto convencional. No concreto com adição de fibras de polietileno de alto módulo, o módulo de elasticidade foi $24,9 \mathrm{GPa}, 7,3 \%$ maior do que no concreto convencional, enquanto concreto com fibras de PP o obteve $22,3 \mathrm{GPa}$, o que significa uma redução de $3,9 \%$ em relação ao concreto sem fibras.

O concreto com adição de fibras de PP apresentou uma resistência à tração média de 4,47 MPA, 9,3\% maior em relação ao concreto convencional, enquanto que o concreto com adição de fibras de polietileno de alto módulo apresentou 4,38 MPa de resistência à tração, 7,1 \% maior do que o concreto sem fibras. As fibras atuaram como reforço na matriz frágil desse material, reduzindo as tensões internas e garantindo assim um melhor desempenho em relação a resistência à tração.

A tenacidade dos concretos reforçados com fibras poliméricas apresentou um aumento significativo em relação ao concreto convencional. O concreto com fibras de polipropileno apresentou $22,27 \mathrm{kN}$.mm de tenacidade, $16,3 \%$ maior do que a tenacidade encontrada para o concreto sem adição de fibras. $\mathrm{O}$ concreto com adição de fibras de HMPE apresentou tenacidade de $24,72 \mathrm{kN}$.mm, o que significa um aumento de $29 \%$ em relação ao concreto sem fibras.

Os resultados deste trabalho demonstram a influência da adição de fibras poliméricas nas propriedades mecânicas do concreto. Não foram encontrados, até o momento da publicação deste trabalho, outros estudos a respeito do uso de fibras de HMPE como adição ao concreto. A única propriedade na qual foi observado influência positiva da adição de fibras poliméricas foi a tenacidade. Nas demais propriedades estudadas neste trabalho a adição de fibras poliméricas não apresentou influência significativa.

\section{AGRADECIMENTOS}

Os autores deste trabalho agradecem ao POSMAT, Curso de Mestrado em Engenharia dos Materiais do CEFET-MG e a CAPES pelo suporte financeiro aos trabalhos realizados. 


\section{BIBLIOGRAFIA}

[1] GUPTA, R.S., Principals of structural design: wood, steel and concrete, 2 ed., Boca Raton, CRC Press, 2014.

[2] BERNARDI, S.T., Avaliação do comportamento de materiais compósitos de matrizes cimentícias reforçadas com fibra de aramida kevlar, Tese de M.Sc., Universidade Federal do Rio Grande do Sul, Porto Alegre, RS, Brasil, 2003.

[3] MACHADO, A.C., SILVA, A.A., FILHO, R.D.T., PFEIL, M.S., LIMA, I., LOPES, R.T., “3D investigation of steel fiber distribution in reinforced concrete by $x$-ray microtomography”, In: Revista Ibracon de Estruturas e Materiais, v. 8, n. 5, pp 707-720, Out. 2015.

[4] SALVADOR, R.P., FERNANDES, J.F., FIGUEIREDO, A.D., "Avaliação do concreto reforçado com baixos teores de fibras para fins estruturais segundo a norma”, Revista Matéria, v. 20, n. 4, pp. 961-974, Dez. 2015.

[5] SALVADOR, R.P., FIGUEIREDO, A.D., "Análise comparativa de comportamento mecânico de concreto reforçado com macrofibra polimérica e com fibra de aço", Revista Matéria, vol.18, n.2, pp. 1273-1285, 2013.

[6] BEZERRA, A.C.S, Concreto com fibras curtas de aço submetido à altas temperaturas, Tese de D.Sc., Universidade Federal de Minas Gerais, MG, Brasil, 2012.

[7] ASLANI, F., SAMALI, B., "High strength polypropylene fibre reinforcement concrete at high temperature", Springer Science and Business Media New York, v. 50, pp. 1229-1247, 2014.

[8] SALVADOR, R.P., Análises comparativas de métodos de ensaio para caracterização do comportamento mecânico de concretos reforçados com fibras, Tese de M.Sc., Escola Politécnica da Universidade de São Paulo, São Paulo, SP, Brasil, 2013.

[9] SUN, Z., XU, Q., "Microscopic, physical and mechanical analysis of polypropylene fiber reinforced concrete”,Materials Science and Engineering A, v. 527, n.1-2, pp. 198-204, Dez. 2009.

[10] LIMA, R.C.A., Investigação do comportamento de concretos em temperaturas elevadas, Tese de D.Sc., Universidade Federal do Rio Grande do Sul, Porto Alegre, RS, Brasil, 2005.

[11] IZAGUIRRE, A., LANAS, J., ALVAREZ, J., I., "Effect of a polypropylene fibre on the bahaviour of aerial lime-based mortars", Construction and Building Materials, v. 25, pp. 992-1000, jun. 2010.

[12] NILI, M., AFROUGHSABET, V., The effects of silica fume and polypropylene fibers on the impact resistance and mechanical properties of concrete", Construction and Building Materials, v. 24, pp. 927-933, nov. 2009.

[13] ZORAN, J., G., GORDANA, A., T., C., NENAD, S., R., IVA M., D., “Abrasion resistance of concrete micro-reinforced with polypropylene fibers", Construction and Building Materials, v. 27, pp. 305-312, Agosto 2011.

[14] KAKOOEI, S., AKIL, H., M., JAMSHIDI, M., ROUHI, J, “ The effects of polypropylene fibers on the properties of reinforced concrete structures", "Construction and Building Materials, v.27, pp. 73-77, Agosto 2011.

[15] SONG, P., S., HWANG, S., SHEU, B., C, "Strength properties of nylon-and polypropylene-fiberreinforced concretes" Cement and Concrete Research, v. 35, pp. 1546-1550, jun. 2004.

[16] BARRERA, G., M., NUÑES, F., U., GENCEL, O., BROSTOW, W., "Mechanical properties of polypropylene-fiber reinforced concrete after gamma irradiation”, Composites: Part A, v. 42, pp. 567-572, jan. 2011.

[17] DAVIES, P., REAUD, Y., DUSSUD, L., WOERTHER, P. "Mechanical behavior of HMPE and aramid fibre ropes for deep sea handling operations", Ocean Engineering, v. 38, n. 17-18, pp. 2208-2214 Dez. 2011.

[18] ASSOCIAÇÃO BRASILEIRA DE NORMAS TÉCNICAS, NBR 8522: Concreto:Determinação do módulo estático de elasticidade à compressão, Rio de Janeiro, 2008.

[19] ASSOCIAÇÃO BRASILEIRA DE NORMAS TÉCNICAS, NBR 12142: Determinação da resistência à tração na flexão em corpos de prova prismáticos, Rio de Janeiro, 2010. 